\title{
Analysis of Compression Ratio of Various Randomly Generated Images of Same Size
}

\author{
Tanvir Prince ${ }^{1}$ \\ ${ }^{1}$ Associate Professor of Mathematics, Hostos Community College, City University of New York, USA \\ Correspondence: Tanvir Prince, Associate Professor of Mathematics, Hostos Community College, City University of \\ New York. USA. E-mail: tprince@ hostos.cuny.edu
}

Received: June 28, 2017 Accepted: July 13, 2017 Online Published: July 19, 2017

doi:10.5539/jmr.v9n4p114 URL: https://doi.org/10.5539/jmr.v9n4p114

\begin{abstract}
Various randomly generated images of size $m \times n$ are created and compressed using the standard TIF compression and their compression ratio are analyzed. TIF compression is used to preserve the original file size. This method is applied for various values of $m$ and $n$. The study of randomized picture in the literature is very rare and this current paper is aimed to fill up that gap.
\end{abstract}

Keyword: randomly generated picture; compression ratio; JPEG; random image

\section{Introduction}

In mathematics, we have studied random path, randomly generated graphs, random matrix etc. But the study of randomly generated images is very rare. The purpose of this paper is to fill this big gap in the literature. We have created random pictures of various sizes of up to 10,000 pixels. Each fixed size is analyzed in two different ways. So for a given image of size, $m \times n$, we consider two sizes $a \times b$ and $c \times d$ so that, $a b=c d=m n$. In each case, 100 random pictures are generated and saved as ".tif" format to preserve original size. We gradually increase $m n$ from 12 to all the way to 10,000 and analyze the file size. We use "Mathematica" software to generate the random image. To learn more about Mathematica, readers are encouraged to see (Purdue University, n.d.) and (Wolfram Mathematica, 2013). All the code is provided in this paper so that the reader can recreate the experiment using a computer.

\section{Various Other Use of Image Compression}

Image compression is an everyday application. When you post an image in the Facebook, or download/upload an image from/to the internet, it automatically goes through an image compression mechanism, most likely, JPEG compression (Group, n.d.), as this being the most standard means of compression. To learn the mathematics behind the JPEG compression see (Image Compression, 2011). Image compression is used in NASA (Center). To learn some specific examples of image compression in NASA, see (Prince, Franco, Salva, \& Windolf, 2014). To learn some modeling with image pixel see (Oyvind, 2007). To learn some other types of image compression, like wavelets image compression, see (Center) and for SVD image compression see (Cooper \& Lorenc, 2006).

\section{Mathematica Code}

We use the following code to create 100 random images of size $m \times n$.

$$
\mathrm{T}=\text { Table[Image[RandomReal }[\{0,1\},\{m, n, 3\}]],\{i, 1,100\}] ;
$$

In the above code, you need to replace the $m$ and $n$ by the appropriate number. Then use the following code to export all the images to a folder:

$$
\begin{aligned}
& \mathrm{P}=\text { Export["C: } \backslash \backslash \text { Users } \backslash \text { prince } \backslash \backslash \text { Google Drive } \backslash \backslash \text { Portfolio_after_promotion } \backslash \backslash \text { publication_after_promotion } \backslash \\
& \backslash \text { Analyse Compression Ratio of Various Randomly Generated Images of Same Size } \backslash \\
& \text { \compare_area_of_12\\
4_by_3\\
image001.tif", T, "VideoFrames"]; }
\end{aligned}
$$

Here everything in red is the "folder path" where you want your images to be saved. The green part tells "Mathematica" to export the images one after the other. Note the .tif extension at the end which means that we are using "TIF" compression. If you want use "PNG" or "JPEG" instead, then use .png and .jpg respectively. 


\section{Sample Data}

Following are some sample of random pictures of various sizes: (in each cases, we have 100 random images)

\section{$12 \times 12$}

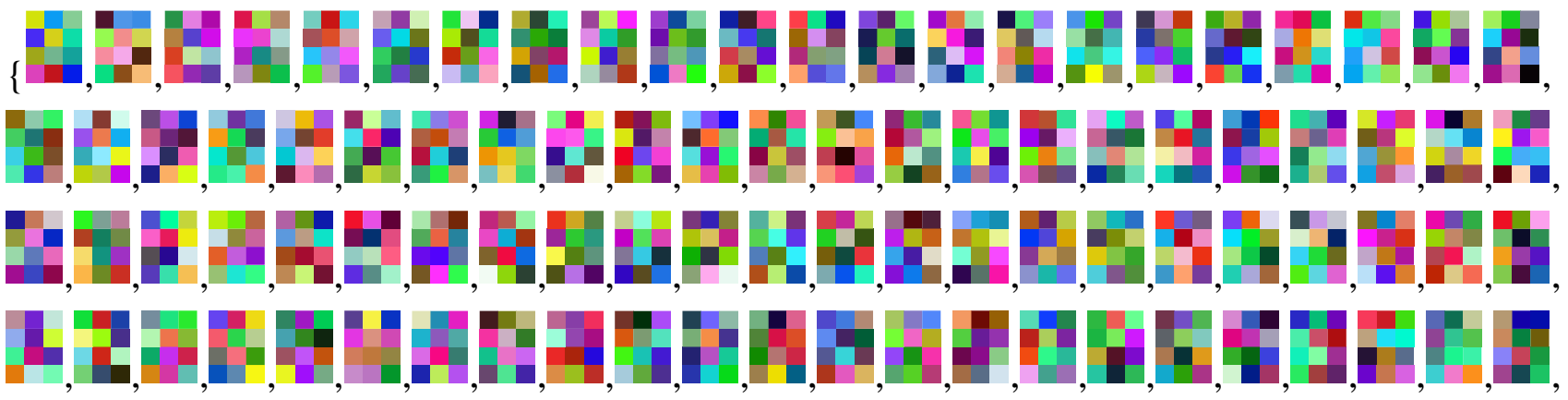

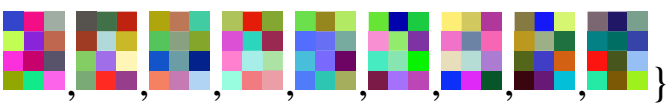

$15 \times 2$

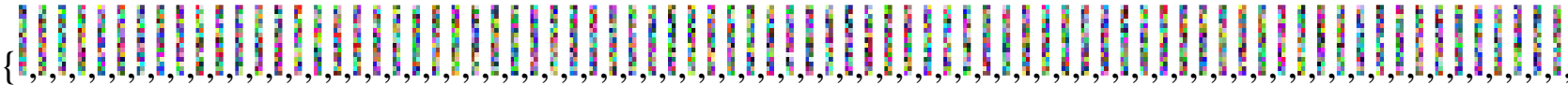

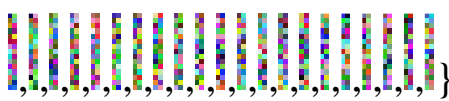

$10 \times 6$

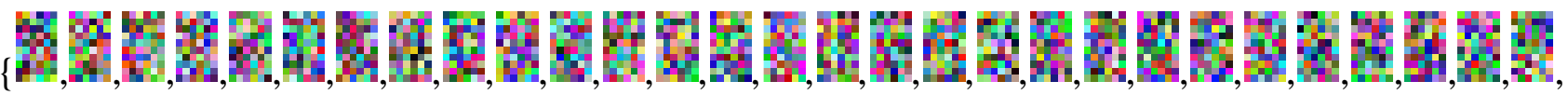

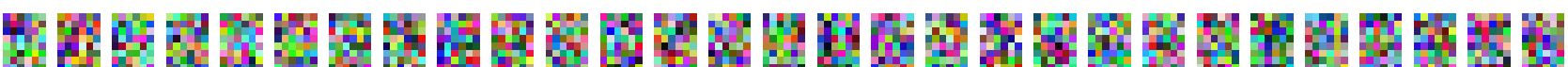

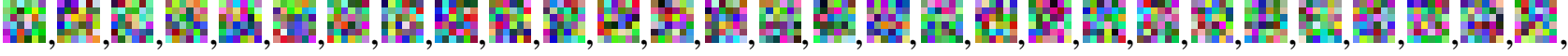

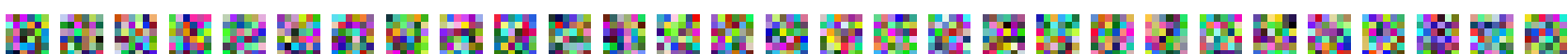
G.

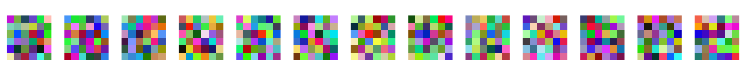

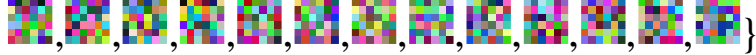

\section{$10 \times 10$}

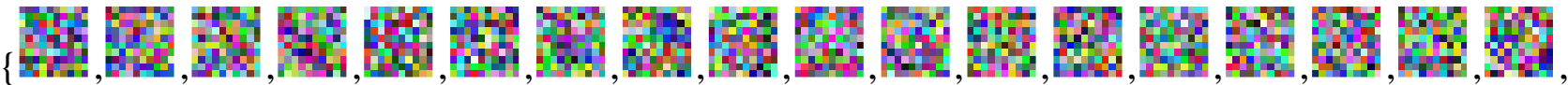

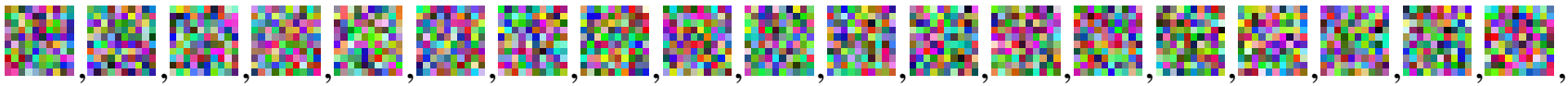
4.

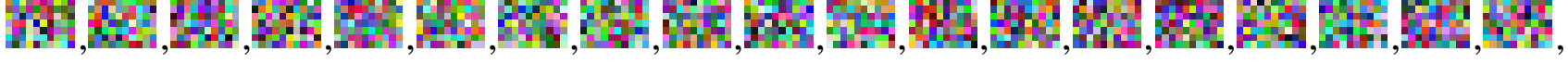

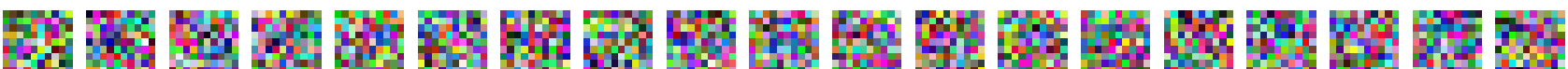

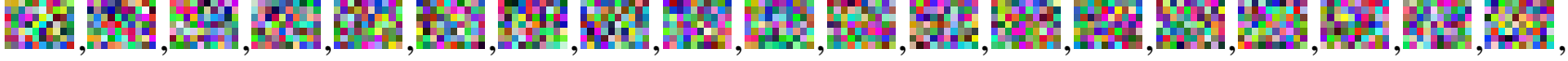

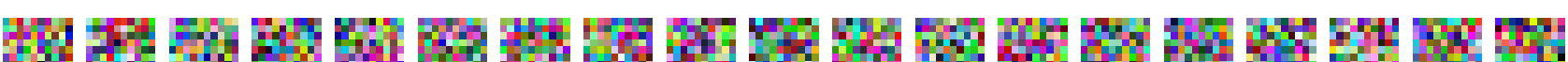

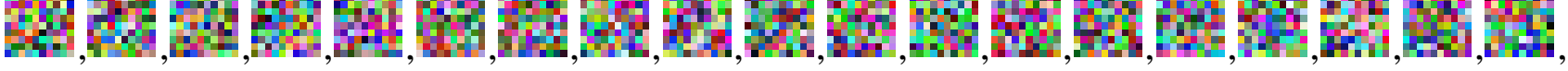

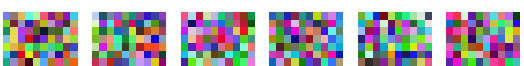

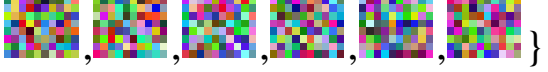




\section{$25 \times 20$}

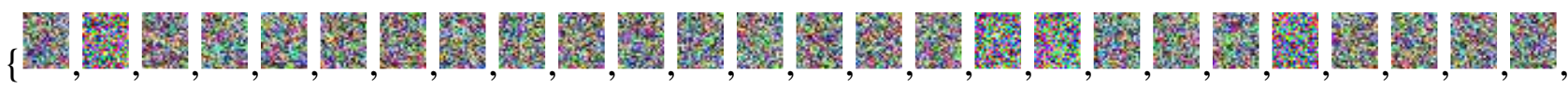

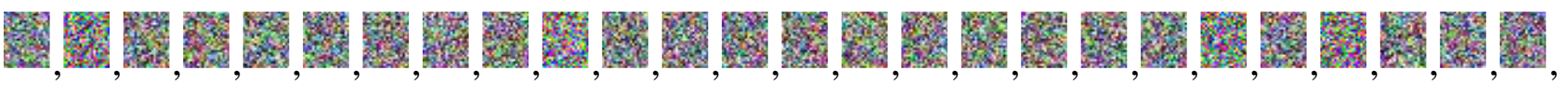

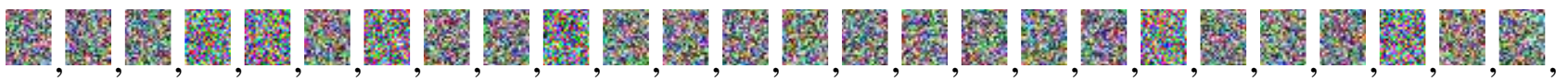

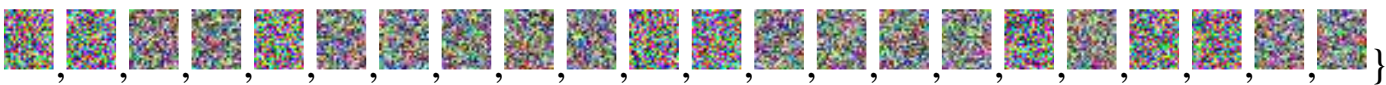

\section{$100 \times 5$}
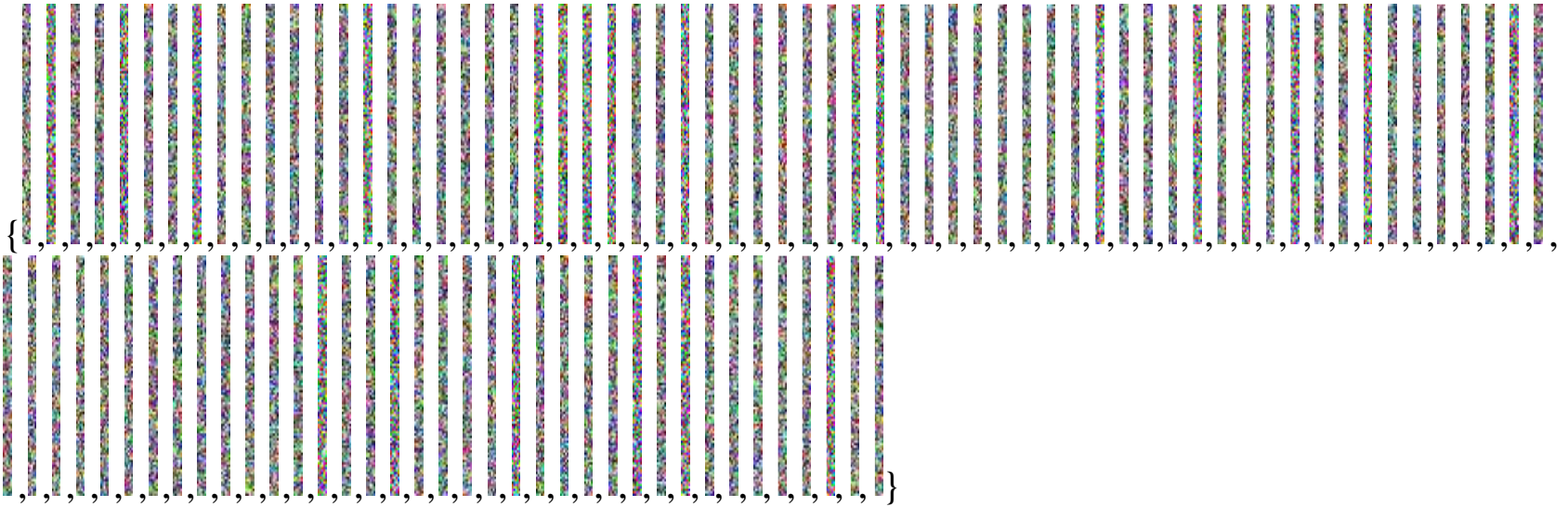

\section{$25 \times 40$}

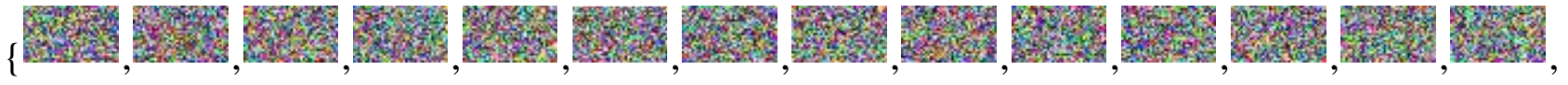

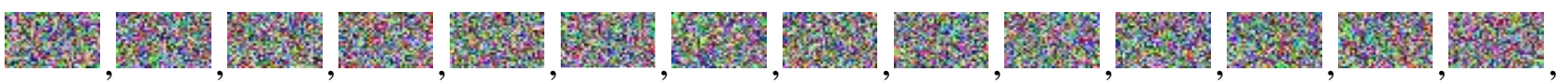
Fing

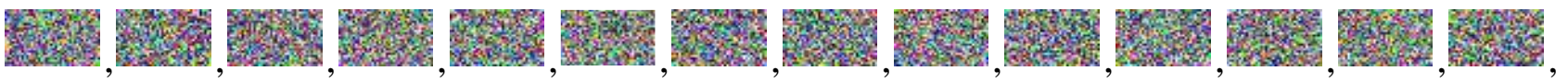

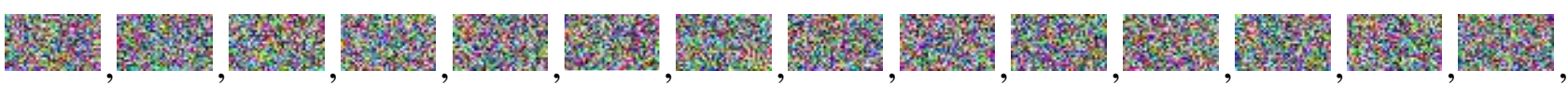

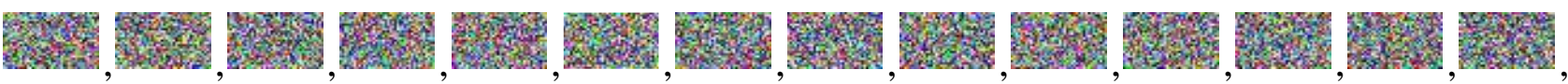

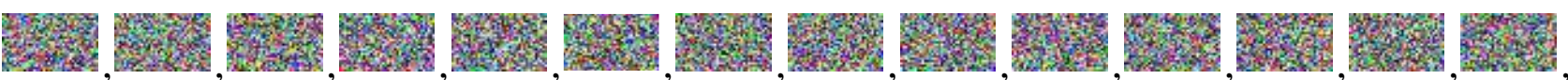
2. 
Sample of Exported File (only a first few are shown) and Their Analysis

\section{$4 \times 3$}

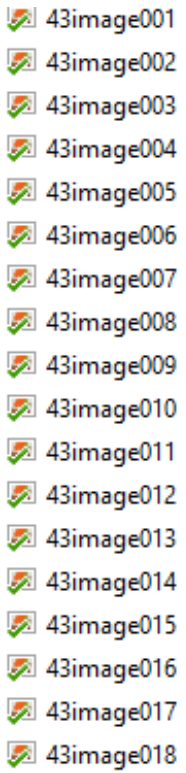

5.7. 62image001

5.: 62image002

b. 62image003

7. 62image004

5: 62image005

5. 62image006

5. 62image007

5. 62image008

5. 62image009

5. 62image010

5. 62image011

5.: 62image012

7) 62image013

5. 62image014

5.7. 62image015

5: 62image016

5. 62image017

5.: 62image018

5. 62image019

5. 62image020

5. 62image021

5. 62image022
6/20/2017 9:42 AM

6/20/2017 9:42 AM

6/20/2017 9:42 AM

6/20/2017 9:42 AM

6/20/2017 9:42 AM

6/20/2017 9:42 AM

6/20/2017 9:42 AM

6/20/2017 9:42 AM

6/20/2017 9:42 AM

6/20/2017 9:42 AM

6/20/2017 9:42 AM

6/20/2017 9:42 AM

6/20/2017 9:42 AM

6/20/2017 9:42 AM

6/20/2017 9:42 AM

6/20/2017 9:42 AM

6/20/2017 9:42 AM

6/20/2017 9:42 AM
TIF File

TIF File

TIF File

TIF File

TIF File

TIF File

TIF File

TIF File

TIF File

TIF File

TIF File

TIF File

TIF File

TIF File

TIF File

TIF File

TIF File

TIF File
$1 \mathrm{~KB}$

$1 \mathrm{~KB}$

$1 \mathrm{~KB}$

$1 \mathrm{~KB}$

$1 \mathrm{~KB}$

$1 \mathrm{~KB}$

$1 \mathrm{~KB}$

$1 \mathrm{~KB}$

$1 \mathrm{~KB}$

$1 \mathrm{~KB}$

$1 \mathrm{~KB}$

$1 \mathrm{~KB}$

$1 \mathrm{~KB}$

$1 \mathrm{~KB}$

$1 \mathrm{~KB}$

$1 \mathrm{~KB}$

$1 \mathrm{~KB}$

$1 \mathrm{~KB}$

\section{$6 \times 2$}

6/20/20179:53 AM TIF File 1 KB

6/20/2017 9:53 AM TIF File $1 \mathrm{~KB}$

6/20/2017 9:53 AM TIF File 1 KB

6/20/2017 9:53 AM TIF File 1 KB

6/20/2017 9:53 AM TIF File $1 \mathrm{~KB}$

6/20/2017 9:53 AM TIF File $1 \mathrm{~KB}$

6/20/2017 9:53 AM TIF File $1 \mathrm{~KB}$

6/20/2017 9:53 AM TIF File $1 \mathrm{~KB}$

6/20/2017 9:53 AM TIF File $1 \mathrm{~KB}$

6/20/2017 9:53 AM TIF File $1 \mathrm{~KB}$

6/20/2017 9:53 AM TIF File 1 KB

6/20/2017 9:53 AM TIF File $1 \mathrm{~KB}$

6/20/2017 9:53 AM TIF File $1 \mathrm{~KB}$

6/20/2017 9:53 AM TIF File 1 KB

6/20/2017 9:53 AM TIF File 1 KB

6/20/2017 9:53 AM TIF File 1 KB

6/20/2017 9:53 AM TIF File 1 KB

6/20/2017 9:53 AM TIF File 1 KB

6/20/2017 9:53 AM TIF File $1 \mathrm{~KB}$

6/20/2017 9:53 AM TIF File $1 \mathrm{~KB}$

6/20/2017 9:53 AM TIF File $1 \mathrm{~KB}$

6/20/2017 9:53 AM TIF File 1 KB

From the above we see that both $4 \times 3$ and $6 \times 2$ takes the same space, $1 \mathrm{~KB}$. 


\section{$10 \times 10$}
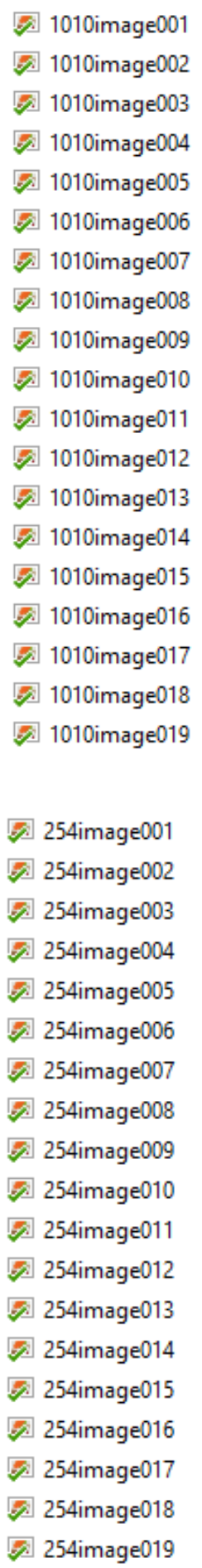

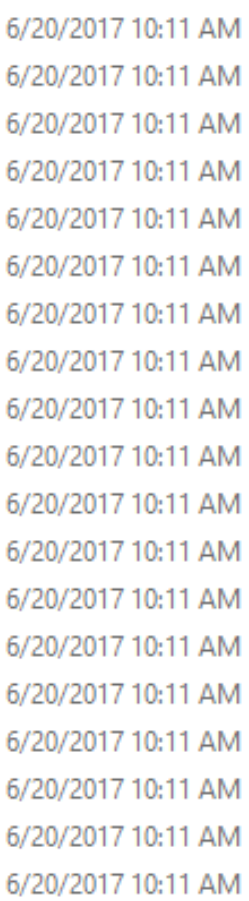

\section{$25 \times 4$}

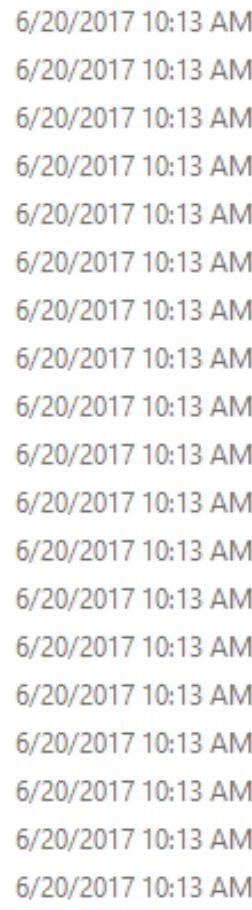

$\begin{array}{ll}\text { TIF File } & 2 \mathrm{~KB} \\ \text { TIF File } & 2 \mathrm{~KB} \\ \text { TIF File } & 2 \mathrm{~KB} \\ \text { TIF File } & 2 \mathrm{~KB} \\ \text { TIF File } & 2 \mathrm{~KB} \\ \text { TIF File } & 2 \mathrm{~KB} \\ \text { TIF File } & 2 \mathrm{~KB} \\ \text { TIF File } & 2 \mathrm{~KB} \\ \text { TIF File } & 2 \mathrm{~KB} \\ \text { TIF File } & 2 \mathrm{~KB} \\ \text { TIF File } & 2 \mathrm{~KB} \\ \text { TIF File } & 2 \mathrm{~KB} \\ \text { TIF File } & 2 \mathrm{~KB} \\ \text { TIF File } & 2 \mathrm{~KB} \\ \text { TIF File } & 2 \mathrm{~KB} \\ \text { TIF File } & 2 \mathrm{~KB} \\ \text { TIF File } & 2 \mathrm{~KB} \\ \text { TIF File } & 2 \mathrm{~KB} \\ \text { TIF File } & 2 \mathrm{~KB}\end{array}$

TIF File $\quad 2 \mathrm{~KB}$

TIF File $2 \mathrm{~KB}$

TIF File $2 \mathrm{~KB}$

TIF File $2 \mathrm{~KB}$

TIF File $2 \mathrm{~KB}$

TIF File $2 \mathrm{~KB}$

TIF File $2 \mathrm{~KB}$

TIF File $2 \mathrm{~KB}$

TIF File $2 \mathrm{~KB}$

TIF File $2 \mathrm{~KB}$

TIF File $2 \mathrm{~KB}$

TIF File $2 \mathrm{~KB}$

TIF File $2 \mathrm{~KB}$

TIF File $2 \mathrm{~KB}$

TIF File $2 \mathrm{~KB}$

TIF File $2 \mathrm{~KB}$

TIF File $2 \mathrm{~KB}$

TIF File $2 \mathrm{~KB}$

TIF File $2 \mathrm{~KB}$

From the above we see that both $10 \times 10$ and $25 \times 4$ takes the same space, $2 \mathrm{~KB}$

\section{$25 \times 20$}




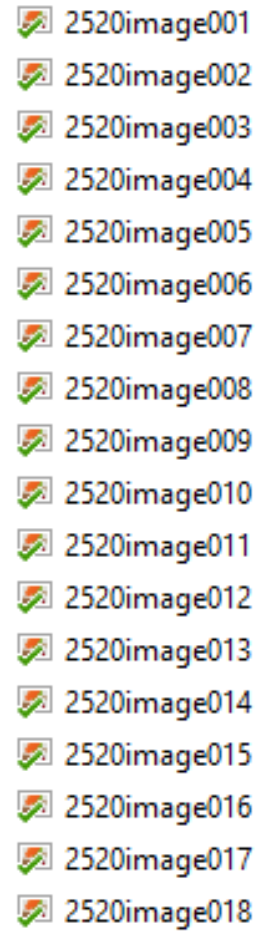

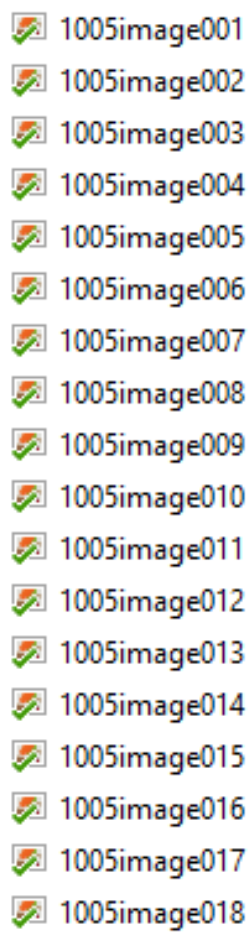

6/20/2017 10:15 AM
6/20/2017 10:15 AM
6/20/2017 10:15 AM
6/20/2017 10:15 AM
6/20/2017 10:15 AM
6/20/2017 10:15 AM
6/20/2017 10:15 AM
6/20/2017 10:15 AM
6/20/2017 10:15 AM
6/20/2017 10:15 AM
6/20/2017 10:15 AM
6/20/2017 10:15 AM
6/20/2017 10:15 AM
6/20/2017 10:15 AM
6/20/2017 10:15 AM
6/20/2017 10:15 AM
6/20/2017 10:15 AM
6/20/2017 10:15 AM

\section{$100 \times 5$}

$\begin{array}{lll}\text { 6/20/2017 10:18 AM } & \text { TIF File } & 4 \mathrm{~KB} \\ \text { 6/20/2017 10:18 AM } & \text { TIF File } & 4 \mathrm{~KB} \\ \text { 6/20/2017 10:18 AM } & \text { TIF File } & 4 \mathrm{~KB} \\ \text { 6/20/2017 10:18 AM } & \text { TIF File } & 4 \mathrm{~KB} \\ \text { 6/20/2017 10:18 AM } & \text { TIF File } & 4 \mathrm{~KB} \\ \text { 6/20/2017 10:18 AM } & \text { TIF File } & 4 \mathrm{~KB} \\ \text { 6/20/2017 10:18 AM } & \text { TIF File } & 4 \mathrm{~KB} \\ \text { 6/20/2017 10:18 AM } & \text { TIF File } & 4 \mathrm{~KB} \\ \text { 6/20/2017 10:18 AM } & \text { TIF File } & 4 \mathrm{~KB} \\ \text { 6/20/2017 10:18 AM } & \text { TIF File } & 4 \mathrm{~KB} \\ \text { 6/20/2017 10:18 AM } & \text { TIF File } & 4 \mathrm{~KB} \\ \text { 6/20/2017 10:18 AM } & \text { TIF File } & 4 \mathrm{~KB} \\ \text { 6/20/2017 10:18 AM } & \text { TIF File } & 4 \mathrm{~KB} \\ \text { 6/20/2017 10:18 AM } & \text { TIF File } & 4 \mathrm{~KB} \\ \text { 6/20/2017 10:18 AM } & \text { TIF File } & 4 \mathrm{~KB} \\ \text { 6/20/2017 10:18 AM } & \text { TIF File } & 4 \mathrm{~KB} \\ \text { 6/20/2017 10:18 AM } & \text { TIF File } & 4 \mathrm{~KB} \\ \text { 6/20/2017 10:18 AM } & \text { TIF File } & 4 \mathrm{~KB}\end{array}$

$4 \mathrm{~KB}$

TIF File 4 KB

TIF File $\quad 4 \mathrm{~KB}$

TIF File $4 \mathrm{~KB}$

TIF File $4 \mathrm{~KB}$

TIF File $4 \mathrm{~KB}$

TIF File $4 \mathrm{~KB}$

TIF File $\quad 4 \mathrm{~KB}$

TIF File $\quad 4 \mathrm{~KB}$

TIF File 4 KB

TIF File $\quad 4 \mathrm{~KB}$

TIF File $\quad 4$ KB

TIF File $\quad 4 \mathrm{~KB}$

TIF File $\quad 4 \mathrm{~KB}$

TIF File $4 \mathrm{~KB}$

TIF File $\quad 4 \mathrm{~KB}$

TIF File $\quad 4 \mathrm{~KB}$

TIF File $4 \mathrm{~KB}$

$4 \mathrm{~KB}$

$\mathrm{KB}$

$4 \mathrm{~KB}$

$4 \mathrm{~KB}$

$\mathrm{KB}$

$4 \mathrm{~KB}$

$\mathrm{KB}$

$\mathrm{KB}$

$4 \mathrm{~KB}$

$\mathrm{KB}$

$\mathrm{KB}$

$4 \mathrm{~KB}$

$\mathrm{KB}$

$\mathrm{KB}$

$\mathrm{KB}$

$\mathrm{KB}$

$4 \mathrm{~KB}$

From the above we see that both $25 \times 20$ and $100 \times 5$ takes the same space, $4 \mathrm{~KB}$ 


\section{$25 \times 40$}
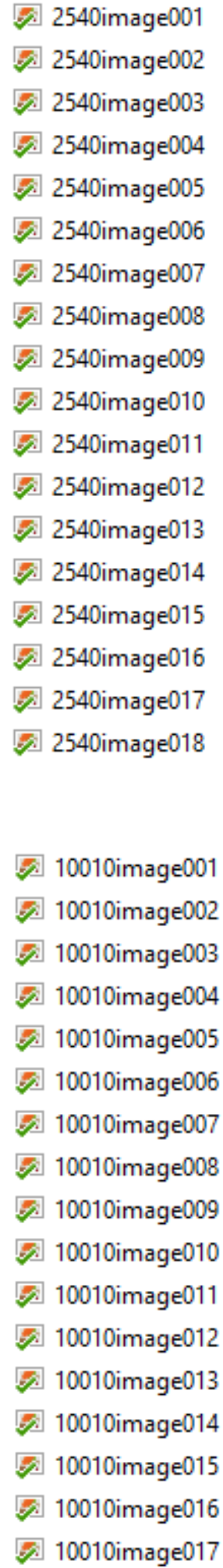

6/20/2017 10:19 AM

6/20/2017 10:19 AM

6/20/2017 10:19 AM

6/20/2017 10:19 AM

6/20/2017 10:19 AM

6/20/2017 10:19 AM

6/20/2017 10:19 AM

6/20/2017 10:19 AM

6/20/2017 10:19 AM

6/20/2017 10:19 AM

6/20/2017 10:19 AM

6/20/2017 10:19 AM

6/20/2017 10:19 AM

6/20/2017 10:19 AM

6/20/2017 10:19 AM

6/20/2017 10:19 AM

6/20/2017 10:19 AM

6/20/2017 10:19 AM

$100 \times 10$

$\begin{array}{ll}\text { TIF File } & 7 \mathrm{~KB} \\ \text { TIF File } & 7 \mathrm{~KB} \\ \text { TIF File } & 7 \mathrm{~KB} \\ \text { TIF File } & 7 \mathrm{~KB} \\ \text { TIF File } & 7 \mathrm{~KB} \\ \text { TIF File } & 7 \mathrm{~KB} \\ \text { TIF File } & 7 \mathrm{~KB} \\ \text { TIF File } & 7 \mathrm{~KB} \\ \text { TIF File } & 7 \mathrm{~KB} \\ \text { TIF File } & 7 \mathrm{~KB} \\ \text { TIF File } & 7 \mathrm{~KB} \\ \text { TIF File } & 7 \mathrm{~KB} \\ \text { TIF File } & 7 \mathrm{~KB} \\ \text { TIF File } & 7 \mathrm{~KB} \\ \text { TIF File } & 7 \mathrm{~KB} \\ \text { TIF File } & 7 \mathrm{~KB} \\ \text { TIF File } & 7 \mathrm{~KB} \\ \text { TIF File } & 7 \mathrm{~KB}\end{array}$

$7 \mathrm{~KB}$

$7 \mathrm{~KB}$

$7 \mathrm{~KB}$

$7 \mathrm{~KB}$

$7 \mathrm{~KB}$

$7 \mathrm{~KB}$

$7 \mathrm{~KB}$

$7 \mathrm{~KB}$

$7 \mathrm{~KB}$

$7 \mathrm{~KB}$

$7 \mathrm{~KB}$

$7 \mathrm{~KB}$

$7 \mathrm{~KB}$

$7 \mathrm{~KB}$

$7 \mathrm{~KB}$

$7 \mathrm{~KB}$

$7 \mathrm{~KB}$

$7 \mathrm{~KB}$

From the above we see that both $25 \times 40$ and $100 \times 10$ takes the same space, $7 \mathrm{~KB}$ 


\section{$100 \times 100$}

\begin{tabular}{|c|c|c|c|}
\hline 100100image001 & 6/20/2017 10:24 AM & TIF File & $60 \mathrm{~KB}$ \\
\hline 100100image002 & 6/20/2017 10:24 AM & TIF File & $60 \mathrm{~KB}$ \\
\hline 100100image003 & 6/20/2017 10:24 AM & TIF File & $60 \mathrm{~KB}$ \\
\hline 100100image004 & 6/20/2017 10:24 AM & TIF File & $60 \mathrm{~KB}$ \\
\hline 100100image005 & 6/20/2017 10:24 AM & TIF File & $60 \mathrm{~KB}$ \\
\hline 100100image006 & 6/20/2017 10:24 AM & TIF File & $60 \mathrm{~KB}$ \\
\hline 100100image007 & 6/20/2017 10:24 AM & TIF File & $60 \mathrm{~KB}$ \\
\hline 100100image008 & 6/20/2017 10:24 AM & TIF File & $60 \mathrm{~KB}$ \\
\hline 100100image009 & 6/20/2017 10:24 AM & TIF File & $60 \mathrm{~KB}$ \\
\hline 100100image010 & 6/20/2017 10:24 AM & TIF File & $60 \mathrm{~KB}$ \\
\hline 100100image011 & 6/20/2017 10:24 AM & TIF File & $60 \mathrm{~KB}$ \\
\hline 100100image012 & 6/20/2017 10:24 AM & TIF File & $60 \mathrm{~KB}$ \\
\hline 100100image013 & 6/20/2017 10:24 AM & TIF File & $60 \mathrm{~KB}$ \\
\hline 100100image014 & 6/20/2017 10:24 AM & TIF File & $60 \mathrm{~KB}$ \\
\hline D. 100100image015 & 6/20/2017 10:24 AM & TIF File & $60 \mathrm{~KB}$ \\
\hline 100100image016 & 6/20/2017 10:24 AM & TIF File & $60 \mathrm{~KB}$ \\
\hline 100100image 017 & 6/20/2017 10:24 AM & TIF File & $60 \mathrm{~KB}$ \\
\hline 100100image018 & 6/20/2017 10:24 AM & TIF File & $60 \mathrm{~KB}$ \\
\hline
\end{tabular}

$200 \times 50$

20050image 001
20050image 002
20050image 003
20050image 004
20050image 005
20050image 006
20050image 007
20050image 008
20050image 009
20050image 010
20050image 011
20050image 012
20050image 013
20050image 014
20050image 015
20050image 016
20050image 017
20050image 018
D. 20050image 019

6/20/2017 10:26 AM
$6 / 20 / 2017$ 10:26 AM
$6 / 20 / 2017$ 10:26 AM
$6 / 20 / 2017$ 10:26 AM
$6 / 20 / 2017$ 10:26 AM
$6 / 20 / 2017$ 10:26 AM
$6 / 20 / 2017$ 10:26 AM
$6 / 20 / 2017$ 10:26 AM
$6 / 20 / 2017$ 10:26 AM
$6 / 20 / 2017$ 10:26 AM
$6 / 20 / 2017$ 10:26 AM
$6 / 20 / 2017$ 10:26 AM
$6 / 20 / 2017$ 10:26 AM
$6 / 20 / 2017$ 10:26 AM
$6 / 20 / 2017$ 10:26 AM
$6 / 20 / 2017$ 10:26 AM
$6 / 20 / 2017$ 10:26 AM
$6 / 20 / 2017$ 10:26 AM
$6 / 20 / 2017$ 10:26 AM

\begin{tabular}{ll} 
TIF File & $60 \mathrm{~KB}$ \\
TIF File & $60 \mathrm{~KB}$ \\
TIF File & $60 \mathrm{~KB}$ \\
TIF File & $60 \mathrm{~KB}$ \\
TIF File & $60 \mathrm{~KB}$ \\
TIF File & $60 \mathrm{~KB}$ \\
TIF File & $60 \mathrm{~KB}$ \\
TIF File & $60 \mathrm{~KB}$ \\
TIF File & $60 \mathrm{~KB}$ \\
TIF File & $60 \mathrm{~KB}$ \\
TIF File & $60 \mathrm{~KB}$ \\
TIF File & $60 \mathrm{~KB}$ \\
TIF File & $60 \mathrm{~KB}$ \\
TIF File & $60 \mathrm{~KB}$ \\
TIF File & $60 \mathrm{~KB}$ \\
TIF File & $60 \mathrm{~KB}$ \\
TIF File & $60 \mathrm{~KB}$ \\
TIF File & $60 \mathrm{~KB}$ \\
TIF File & $60 \mathrm{~KB}$ \\
\hline
\end{tabular}

From the above we see that both $100 \times 100$ and $200 \times 50$ takes the same space, $60 \mathrm{~KB}$

\section{Conclusion}

So we conclude that the space of the images of size $a \times b$ and $c \times d$ is same as long as $a b=c d$. But as the product, $m n$, increase for the image of size $m \times n$, the space will also increase. We also notice that as we increase the product $m n$, 10 times, the space does not increase 10 times - it actually increases less. For example, when $m n$ increase from 1,000 to 10,000 , the space increase from $7 \mathrm{~KB}$ to $60 \mathrm{~KB}$ (a little less than 10 times). In this experiment, we only save the output 
image as "TIF" format. The readers are encouraged to redo the experiment and use different types of output format, for example, "PNG" and "JPEG" etc. and see how the result changes, if any.

\section{References}

Center, N. A. (n.d.). Perceptual Optimization of Wavelet Image Compression. (n.d.). Department of Electrical Engineering, UCLA.

College, H. M. (n.d.). Change of Basis. Retrieved from Math Totorial Website: http://www.math.hmc.edu/calculus/tutorials/changebasis/changebasis.pdf

Cooper, I., \& Lorenc, C. (2006). Image Compression Using Singular Value Decomposition. Retrieved from Retrieved from College of the Redwoods: http://msemac.redwoods.edu/ darnold/math45/laproj/fall2006/iancraig/SVD_paper.pdf

George, B. Thomas, J. (2004). Thomas Calculus Early Transcendentals. Addison-Wesely, earson.

Group, S. (n.d.). JPEG Compression: What it is - when to use it - and when not to. Retrieved from Retrieved from the university of Oslo website: http://folk.uio.no/inf9540/SVD.pdf https://www.spacetelescope.org/projects/fits_liberator/improc/

Image Compression. (2011). Image Compression: How Math Led to the JPEG2000 Standard. Retrieved from Image Compression: How Math Led to the JPEG2000 Standard. (2011).

Retriwww.whydomath.org/node/wavlets/basicjpg.html

Math is Fun. (n.d.). Retrieved from http://www.mathsisfun.com/divisibility-rules.html

Mathematics, S. f. (2011). Why Do Math? Retrieved from Image Compression: How Math Led to the JPEG2000 Standard: http://www.whydomath.org/node/wavlets/basicjpg.html

Nielsen. (n.d.). Nielsen data analysis for media. Retrieved from http://www.nielsen.com/us/en.html

Oyvind, K. (2007). Introduction to Pixel Modeling. Retrieved from Image Processing with Glaus: www.pippin.gimp.org/image_processing

Prince, T. (2012, May). Using Youtube in Calculus I to Increase Class Participation. Mathematics Teaching Research Journal Online, 5(2), 64-69. Retrieved from http://www.hostos.cuny.edu/departments/math/MTRJ/index.htm

Prince, T. (2012, September). Online or Offline? International Journal of Mathematical Archive, 3(9), 3374-3379. Retrieved from www.ijma.info

Prince, T. (2013, May). Kaprekar Constant Revisited. International Journal of Mathematical Archive, 4(5), 1-7. Retrieved from www.ijma.info

Prince, T. (January 13-14, 2013). The Use of Online Videos in Blackboard Platform: Use of Pre-Lecture Videos in Calculus. Ninth International Conference on Technology, Knowledge and Society. Vancouver, Canada. Retrieved from http://youtu.be/T79knc-jO8o

Prince, T. (July 9, 2012). Using Online Videos to Increase Class Participation. In H. C. College (Ed.), The International Learning Style Conference. New York.

Prince, T. (March 9, 2013). Online or Offline? Tristate Best Practices Conference: The Community Colleges as a Place of Transition. Meadowlands, New Jersey: Bergen Community College. Retrieved from http://www.bergen.edu/pages1/pages/Best-Practices-Conference.aspx

Prince, T. (May 3, 2013). Flipping Over Math: Computer Based Homework and Quizzes for Non-Traditional Students. Bronx EdTech Showcase, 2013. New York: Lehman College, CUNY. Retrieved from http://bronxtech2013.commons.gc.cuny.edu/

Prince, T., \& Nieves, A. (January 3-5, 2013). Encouraging and Motivating Minority Engineering Students Through Summer Research Initiative. 2013 Maui International Engineering Education Conference. Maui, Hawaii: The Clute Institute. Retrieved from http://www.cluteinstitute.com/proceedings/2013HIProceedings.html

Prince, T., \& Nieves, A. (January 6-9, 2013). Encouraging and Motivating Minority Engineering Students Through Summer Research Initiative. 11th Annual Hawaii International Conference on Education. Honolulu, Hawaii: Hawaii International Conference on Education. Retrieved from http://www.hiceducation.org/program_edu.php

Prince, T., Franco, S., Salva, I., \& Windolf, C. (2014). Mathematics Behind Image Compression. Journal of Student Research, 3(1), 46-62. Retrieved from http://www.jofsr.com/index.php/path

Purdue University. (n.d.). A Brief Introduction to Mathematica. Retrieved from 
http://www.cs.purdue.edu/homes/ayg/CS590C/www/mathematica/math.html

Rahman Z., J. D. (n.d.). Image enhancement, image quality, and noise, Photonic Devices and Algorithms for Computing., (pp. VII, Proc. SPIE 5907 ).

Science, R. S. (n.d.). Department of Mathematics Website. Retrieved from http://www.math.rutgers.edu/undergrad/Activities/drp/

Seaman, R. P. (2006). Astronomical Tiled Image Compression: How and Why. . ASP Conference Series, 1-4.

Seaman, R., Pence, W., White, R., Dickinson, M., Valdes, R., \& Zarate, N. (2006). Astronomical Tiled Image Compression: How and Why. Astronomical data analysis software and systems, XVI.

Silverman, J. H. (2012). A Friendly Introduction to Number Theory (4th ed.).

Space, N. A. (n.d.). National Aeronautics and Space Administration. Retrieved from National Aeronautics and Space Administration: www.nasa.gov

Spacetelescope. (2013). Spacetelescope.org. Retrieved from Spacetelescope.org:

Steedly, K., Dragoo, K., Arafeh, S., \& Luke, S. D. (2008). Effective Mathematics Education. Evidence for Education, III(I).

Stewart, G. W. (1993). On The Early History of the Singular Value Decomposition.

Stewart, J. (2012). Stewart Calculus Early Transcendentals 8th Edition. Cengage Learning.

Strang, G. (2009). Introduction to Linear Algebra (4th ed.). Wellesley-Cambridge Press.

White, W. A. (n.d.). Data Compression for Full Motion Video Transmission. Conference on Advanced Space Exploration Initiative Technologies, 1-11.

wikipedia. (2010). Retrieved from wikipedia.org: http://en.wikipedia.org/wiki/Motivation

Wikipedia. (n.d.). Polydivisible Numbers. Retrieved from The Free Encyclopedia: http://en.wikipedia.org/wiki/Polydivisible_number

Wolfram Mathematica. (2013). Hands-on Start to Mathematica. Retrieved from Wolfram: http://www.wolfram.com/broadcast/screencasts/handsonstart/

\section{Copyrights}

Copyright for this article is retained by the author(s), with first publication rights granted to the journal.

This is an open-access article distributed under the terms and conditions of the Creative Commons Attribution license (http://creativecommons.org/licenses/by/4.0/). 